\title{
Modelo estocástico de la transmisión de enfermedades infecciosas
}

\author{
Juan Ruiz-Ramírez, MC, DC, ${ }^{(1,2)}$ Gabriela Eréndira Hernández-Rodríguez, L Econ. ${ }^{(1)}$
}

\begin{abstract}
Ruiz-Ramírez J, Hernández-Rodríguez GE. Modelo estocástico de la transmisión de enfermedades infecciosas. Salud Publica Mex 2009;5 1:390-396.
\end{abstract}

\begin{abstract}
Resumen
Objetivo. Proponer un modelo estocástico que muestre la forma en que afecta la estructura de la población al tamaño de la epidemia de las enfermedades infecciosas. Material y métodos. Este estudio se realizó en la Universidad de Colima en el año 2004. Se utilizó la topología de red del mundo pequeño generalizada para representar los contactos ocurridos dentro y entre familias; para ello se realizaron dos programas en MATLAB para calcular la eficiencia de la red; también se requirió la elaboración de un programa en el lenguaje $C$, que representa el modelo estocástico susceptible-infecciosoremovido, y se obtuvieron resultados simultáneos del número de personas infectadas. Resultados. El incremento del número de familias conectadas por los sitios de reunión modificó el tamaño de las enfermedades infecciosas en una proporción de casi $400 \%$. Discusión. La estructura de la población influye en la propagación rápida de las enfermedades infecciosas y puede alcanzar efectos epidémicos.
\end{abstract}

Palabras clave: enfermedades transmisibles; modelos matemáticos; redes; México
Ruiz-Ramírez J, Hernández-Rodríguez GE. Stochastic model of infectious diseases transmission.

Salud Publica Mex 2009;5 1:390-396.

\section{Abstract}

Objective. Propose a mathematic model that shows how population structure affects the size of infectious disease epidemics. Material and Methods. This study was conducted during 2004 at the University of Colima. It used generalized small-world network topology to represent contacts that occurred within and between families. To that end, two programs in MATLAB were conducted to calculate the efficiency of the network. The development of a program in the $C$ programming language was also required, that represents the stochastic susceptible-infectious-removed model, and simultaneous results were obtained for the number of infected people. Results. An increased number of families connected by meeting sites impacted the size of the infectious diseases by roughly $400 \%$. Discussion. Population structure influences the rapid spread of infectious diseases, reaching epidemic effects.

Key words: communicable diseases; mathematical models; network; Mexico

(I) Facultad de Economía. Universidad Veracruzana. Xalapa,Veracruz, México.

(2) Centro Virtual de Investigaciones Multidisciplinarias (CEVIM), Universidad Veracruzana. Xalapa, Veracruz, México.

Fecha de recibido: 9 de octubre de 2008 - Fecha de aprobado: 17 de junio de 2009 Solicitud de sobretiros: Mtro. Juan Ruiz Ramírez. Universidad Veracruzana. Av. Xalapa s/n, esq. Manuel Ávila Camacho. 91020 , Xalapa, Veracruz, México. Correo electrónico: jruizuv@gmail.com 
L as enfermedades infecciosas representan un verdadero problema de dimensiones globales en la salud pública, debido a que provocan alrededor de 13 millones de muertes cada año, ${ }^{1}$ es decir, son la principal causa de mortalidad en el ámbito mundial y la tercera en Estados Unidos de América. ${ }^{2}$

Según Kay, ${ }^{3}$ la Organización Mundial de la Salud (OMS) indica que las enfermedades infecciosas produjeron 14.7 millones de muertes en el año 2001, lo que corresponde a $26 \%$ de la mortalidad global. Entre ellas figuran el síndrome de inmunodeficiencia adquirida (VIH/sida), la tuberculosis y la malaria, que ocasionaron 2.9, 1.6 y 1.1 millones de decesos, respectivamente, lo cual equivale a $38 \%$ de las defunciones consecutivas a enfermedades infecciosas ese año.

En la actualidad se dispone de fármacos y vacunas que ayudan a prevenir muchas muertes por enfermedades infecciosas. Además, se evitarían casi dos millones de decesos ${ }^{3}$ si se educara a la población para evitar el consumo de agua fecalmente contaminada.

En el estudio de las enfermedades infecciosas se utilizan modelos matemático ${ }^{4,5}$ que describen su evolución; tales modelos representan situaciones que sólo difícilmente se obtendrían mediante observación directa o a través de experimentación. ${ }^{6}$

El objetivo de los modelos matemáticos aplicados a la epidemiología es proporcionar información útil para tomar decisiones, establecer medidas operativas en el control o erradicar una enfermedad infecciosa. ${ }^{7-9}$

Dichos modelos se pueden clasificar en determinísticos y estocásticos. Los primeros son relativamente sencillos, ya que resultan de representar un fenómeno biológico a través de la resolución de ecuaciones diferenciales. Los segundos incluyen el término aleatorio de diversas formas. ${ }^{7}$

En una población, las personas susceptibles a una enfermedad infecciosa comúnmente se dividen en pequeños grupos que interactúan de manera más estrecha, por ejemplo familias, grupos de alumnos, etc. $\mathrm{Al}$ considerar que los integrantes de las familias interactúan entre sí, es posible que se incremente la tasa de contactos. Los miembros de un grupo interactúan con uno o más miembros de otros grupos, como en la escuela, el cine, el trabajo, los centros de diversión, etc., definidos como sitios de reunión, lo que posibilita que la enfermedad trascienda entre ellos y avance en la población. ${ }^{10}$

Un ejemplo de la importancia de los sitios de reunión lo presentaron Longini y Koopman, ${ }^{10}$ en cuya aplicación de los modelos matemáticos determinaron que las escuelas se convirtieron en un foco de infección para la enfermedad de la influenza. Esta información la utilizó el gobierno de Hong Kong para inmunizar contra la influenza a niños que asistían a escuelas, lo cual mostró eficacia en el control de la enfermedad.

En el estudio del sarampión, las familias y las escuelas casi nunca se incluyen en los modelos estocásticos que pueden influir en la sobreestimación de la incidencia de este padecimiento. ${ }^{11}$

Otra situación se presentó al estudiar a las familias mediante modelos matemáticos en la vacunación contra la influenza. La medida óptima consistió en vacunar a todos los integrantes (cuatro, cinco, seis y siete miembros); no obstante, es menos eficiente la vacunación en familias de tamaño pequeño. ${ }^{5}$

En los modelos matemáticos se utiliza un parámetro importante: la tasa de reproducción básica $\left(\mathrm{R}_{0}\right)$, definida como el número promedio de contactos efectivos realizados por una persona infectada durante su periodo completo de infección, cuando se introduce en una población susceptible. El valor de este parámetro proporciona información acerca del tamaño de un brote epidemiológico y se emplea para conocer el umbral de la epidemia. $\mathrm{R}_{0}$ se relaciona con el número de infectados al terminar una epidemia, lo que se conoce como el tamaño final de la epidemia. ${ }^{12-17}$

La diferencia entre un modelo determinístico y uno estocástico se manifiesta en el efecto de $R_{0}$ en el modelo: en un modelo determinístico, si $\mathrm{R}_{0}$ es menor de 1 , la enfermedad es endémica y tiende a desaparecer, pero si es mayor de 1, entonces se presenta una epidemia. En cambio, en el modelo estocástico, si $R_{0}$ es mayor de 1 , es probable que ocurra una epidemia. ${ }^{18}$

La dinámica de la transmisión de las enfermedades infecciosas se representa a través de los principales modelos epidemiológicos: susceptible-infeccioso (S-I), susceptible-infeccioso-susceptible (S-I-S), susceptibleinfeccioso-removido (S-I-R) y susceptible-infecciosoremovido-susceptible (S-I-R-S), ${ }^{5,19}$

El modelo más común es el S-I-R, que se utiliza en enfermedades infecciosas de ciclo corto en las que se adquiere inmunidad permanente después de padecer la infección, ${ }^{20}$ como ocurre con la rubéola, el sarampión, la varicela, las infecciones virales y el dengue.

El principal problema que se presenta en la mayor parte de los modelos matemáticos es que ignoran la estructura de la población, factor importante en la diseminación de la enfermedad infecciosa. ${ }^{21}$ Lo sobresaliente de estudiar la estructura de la población a través de redes radica en que si éstas se encuentran muy conectadas, entonces se incrementa el riesgo de transmitir enfermedades virulentas y parasitarias. ${ }^{22}$

Las redes están compuestas por nodos o vértices conectados por ligas y abarcan los siguientes tipos: sociales, ${ }^{23}$ económicos, ${ }^{24}$ neuronales, ${ }^{25}$ computacionales, ${ }^{26}$ 
por citar sólo algunos ejemplos, en los cuales los nodos o vértices se representan en un círculo a través de puntos equidistantes, que se traducen en los elementos del sistema: personas, empresas, neuronas, terminales de computadoras, etc. Por su parte, las ligas que los unen representan las interacciones entre ellos, por ejemplo amistades, transacciones comerciales, nervios, redes que se comunican con las computadoras, etcétera. ${ }^{27,28}$

En las redes se describe la dinámica de la población a través de los patrones de contacto. La importancia de estudiarlos en una población de susceptibles reside en el hecho de que determina el riesgo de contraer una infección. ${ }^{9}$ Éstos influyen de manera significativa en la dispersión de una enfermedad infecciosa, motivo por el cual se considera que los contactos se realizan localmente y a distancia. ${ }^{23,29,30}$

La interrogante que plantean Fell y Wagner ${ }^{31}$ es la siguiente: “... ¿Cómo las redes que son grandes y dispersas pueden, a pesar de eso, ser recorridas en muy pocas etapas o con muy pocas ligas?". Al respecto, Watts y Strogatz ${ }^{32}$ determinaron que la red del mundo pequeño es extremadamente eficiente. Estas redes encuentran por lo regular la vía más corta entre cualquier par de vértices. 33

Existe la teoría de red del mundo pequeño (RMP), según la cual para establecer contacto con otras personas en la transmisión de una enfermedad infecciosa, mensaje o señal, no es necesario conocer a todos los miembros de la población, si bien se requiere interacción eficiente entre ellos. ${ }^{32,34-36}$ En esta RMP se desconectan algunos vértices cercanos para conectarlos de manera aleatoria con algunos otros.

En una red regular se representan los contactos dentro de las familias, los vértices son las $\mathrm{N}$ personas de la población y cada uno se conecta a través de $\mathrm{k}$ ligas, ${ }^{24}$ éstas representan los contactos entre ellos y una infección sólo se puede diseminar a través de las ligas ${ }^{19}$ que unen a cada vértice con sus $\mathrm{k} / 2$ vértices vecinos localizados a los lados.

Una red regular se utiliza cuando se efectúan contactos dentro de las casas (contactos locales) y una red del mundo pequeño es útil cuando en la red regular se desconectan algunos vértices, de tal manera que las ligas eliminadas se usan para unir parejas de vértices de forma aleatoria. Por lo general se agrega una baja proporción de ligas y se espera que el tamaño de la red disminuya de forma drástica. ${ }^{26}$ Estas ligas adicionales representan los contactos entre los sitios de reunión (contacto global). En la figura 1 se muestra la red del mundo pequeño generalizada (RMPG), en la cual no se realizan cortes para desconectar a un vértice de su vecino más cercano ${ }^{26}$ para conectarse con otros vértices lejanos, a diferencia de la red del mundo pequeño.

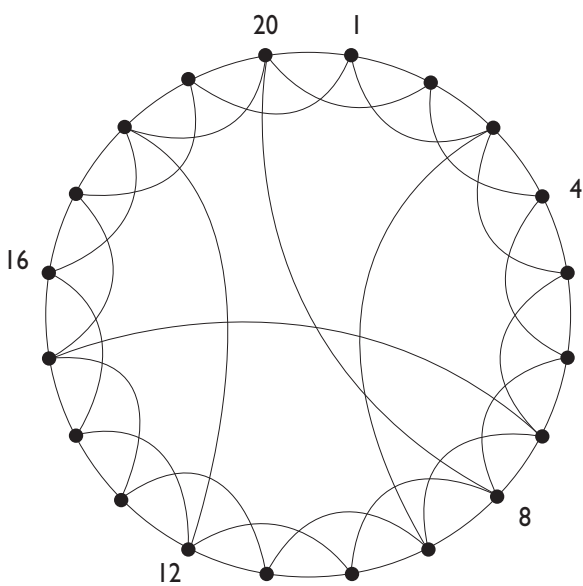

Figura I. Red con sitios de reunión CON 20 vértices Y cuatro ligas. Colima, México, 2004.

En las topologías de redes se consideran dos propiedades. La primera consiste en el promedio mínimo de las ligas necesarias para conectar aleatoriamente a cualquier par de vértices, lo que se conoce como el tamaño de la red (L). ${ }^{19,37}$ La segunda propiedad es el coeficiente de agrupación $(\mathrm{C})$, que expresa la proporción de interacciones que realizan entre sílos vértices unidos a uno en particular. ${ }^{37}$

Una red es eficiente cuando L es pequeño, aproximadamente menor o igual a 4; esto indica que se necesitan en promedio cuatro ligas para unir de manera aleatoria cualquier par de vértices, aunque se han encontrado redes con valores que fluctúan entre 4 y 9.28 Los valores de $\mathrm{C}$ notificados son casi iguales a 0.5 . La red del pundo pequeño tiene un valor bajo de $\mathrm{L} y$ alto de C; esto señala elevada interacción en la red. ${ }^{38,39}$

La estructura de la red del mundo pequeño proporciona mejores resultados ${ }^{40} \mathrm{o}$ se aproxima en eficiencia a la red aleatoria en la que se realizan múltiples contactos entre los vértices, las también conocidas mezclas homogéneas. ${ }^{41,42}$ Por lo anterior, se plantea el objetivo de "Proponer un modelo estocástico que muestre cómo afecta la estructura de la población al tamaño de la epidemia de una enfermedad infecciosa".

\section{Material y métodos}

Para calcular el tamaño de la red y el coeficiente de agrupación de la RMPG se efectuaron dos programas computacionales en MATLAB. ${ }^{*}$ El primero calcula la

\footnotetext{
* Etter D. Introduction to MATLAB for Engineers and Scientists. EUA: Editorial Prentice-Hall, 1996.
} 
matriz de contactos $(\mathrm{P})$ que representa la conexión de los vértices en la red, donde $P_{i, j}=1$ si los vértices i y $j$ están conectados, y es 0 de otra manera. ${ }^{26,27,37} \mathrm{El}$ segundo programa calcula Ly C.

En el modelo estocástico S-I-R, cada individuo infeccioso tiene contactos con otros seleccionados aleatoriamente, con una tasa $\lambda$. El objetivo de un contacto es cualquier actividad que resulta en la infección de un susceptible a través de un individuo infeccioso. ${ }^{43}$

Una vez que un sujeto permanece infectado, se recupera con una tasa $\mu$. Las tasas $\lambda$ y $\mu$ se asumen como parámetros de distribuciones exponenciales, lo cual puede representarse por medio de un proceso de Poisson para ambos eventos: infeccioso y recuperado.

En este trabajo se asume una población de tamaño constante $\mathrm{N}$ y entonces $\mathrm{N}=\mathrm{I}+\mathrm{S}+\mathrm{R}$. Los estados de un proceso en el tiempo t pueden identificarse por $X(t)$ $=\{\mathrm{I}, \mathrm{R}\}$; esto es, el número de individuos infectados $\mathrm{y}$ recuperados en el tiempo t. Cuando hay I infectados y $S$ susceptibles, la tasa de infección es $\lambda \mathrm{IS} / \mathrm{N}$ y la de recuperación es $\mu \mathrm{I}$.

La variable aleatoria $X$ con parámetros $\mu / \lambda+\mu$ se define como "El número de contactos que realiza un individuo infectivo durante su completa vida infecciosa". En un modelo S-I-R, la epidemia se detiene cuando sólo hay dos clases de individuos en la población: los recuperados o inmunes y los susceptibles. El número de individuos infectados denomina el tamaño de la epidemia. Éste puede ser aproximado ${ }^{44}$ con la solución de la ecuación 1.

$$
\mathrm{x}=\mathrm{N}\left(1-\mathrm{e}^{-\mathrm{x} R 0 / \mathrm{N}}\right)
$$

Al sustituir, en la expresión anterior, los valores de $\mathrm{R}_{0}$ y el tamaño de la población $\mathrm{N}$, con el programa Mathematica, se calculó el tamaño de la epidemia (X) bajo el supuesto de mezclas homogéneas y contactos efectivos.

Para estimar el tamaño de la epidemia se elaboró el programaSIMULAMPG en el lenguaje de programación "C", el cual utilizó el modelo estocástico que consideró la topología de la RMPG y el modelo S-I-R.

En la simulación o representación de una epidemia se ejecuta el programa SIMULAMPG para conocer el número promedio de infectados una vez que la epidemia concluyó. La mayoría de los programas de simulación realiza entre 30 y 5000 simulaciones. ${ }^{26,41}$ En este trabajo se realizaron 1000 simulaciones, equivalentes a 1000 epidemias. ${ }^{4}$

En la ejecución del programa de simulación se selecciona el ícono SIMULAMPG y se le ingresa la siguiente información: número de familias; número de integrantes; valor del parámetro $\mu$; valor del parámetro $\lambda$; número de simulaciones y nombre del archivo para almacenar en los resultados.

En este trabajo se estimó $\mathrm{R}_{0}$ mediante el cociente $\lambda / \mu$, que equivale a la tasa de recuperación dividido entre la tasa de infección. ${ }^{22}$

El tamaño de la epidemia esperado se obtuvo cuando $\mathrm{r}$ familias fueron conectadas, mediante el promedio del número de personas infectadas, obtenido en 1000 simulaciones. En cada simulación se conectaron aleatoriamente a r familias, para evitar el efecto de una topología fija, como es el caso de una red regular.

Cada dato obtenido correspondió al tamaño de la epidemia, relacionado con el número de familias conectadas $\left(\mathrm{n}_{\mathrm{f}}\right)$ y su correspondiente número de simulación. Esa información dificultó conocer el comportamiento o tendencia del tamaño de la epidemia y por ello se realizó lo siguiente:

- En el programa MATLAB se importaron los resultados de las simulaciones y se generó la matriz transpuesta, tras crear un nuevo archivo en formato ASCII con la finalidad de facilitar su análisis y tener una matriz con 1000 renglones y $n_{f}$ columnas.

- En el programa computacional Statistica* para Windows, versión 6.0, se utilizó el módulo de estadística descriptiva para calcular el tamaño promedio de la epidemia a través de la media aritmética, y mediante gráficos se describió la tendencia de las epidemias que permitieron compararla con diferentes valores de $R_{0}$ número de familias conectadas, así como distintos tamaños. ${ }^{44}$

Para comparar el tamaño de la epidemia esperado, cuando $\mathrm{r}$ familias se conectan, en relación con mezclas aleatorias, obtenidas por la ecuación (1), se obtuvo el índice $\mathrm{V}_{\mathrm{r}}$ donde:

$$
V_{r}=\frac{\text { Tamaño de la epidemia esperado cuando } r \text { familias son conectadas }}{\text { Tamaño de la epidemia esperado bajo mezclas homogéneas }}
$$

\section{Resultados}

Los resultados más relevantes que se obtuvieron corresponden a la topología de red y del tamaño final de la epidemia, con el fin de mostrar cómo los sitios de reunión influyen en la eficiencia de la red ${ }^{27}$ y en la diseminación de una enfermedad infecciosa. ${ }^{9}$

En la figura 2 se muestra el comportamiento de una epidemia de una población de 250 personas, cuyo tamaño fluctuó entre $0(0 \%)$ y $115(46 \%)$ personas infectadas.

\footnotetext{
* McCallum B. A carnival of stats. Science 1999;284:1291-1292.
} 
Se observa que al aumentar $\mathrm{R}_{0} \mathrm{y}$ el número de familias conectadas se incrementa el tamaño de la epidemia hasta $400 \%$, al comparar cuando no interactúa ninguna familia, respecto de cuando interactúan todas.

El comportamiento de $\mathrm{Ly} \mathrm{C}$, así como el del cociente $\mathrm{V}_{\mathrm{r}}$ se presentan en la figura 3 , cuando $\mathrm{k}$ es igual a cuatro ligas por vértice. El lado izquierdo del eje vertical corresponde a los valores de L. En el lado derecho se presentan las proporciones.

En la figura 3 se observa que el valor de $C$ se encuentra en alrededor de 0.5 , mientras que el valor de $L$ decrece rápidamente en relación con la interacción de $\mathrm{r}$ familias y el tamaño de la red puede alcanzar valores de 4.

El coeficiente $\mathrm{V}_{\mathrm{r}}$ se aproxima a $30 \%$. El valor máximo se alcanzó cuando se conectaron todas las familias con una $\mathrm{R}_{0}$ de cinco. También se observó que la red del mundo pequeño generalizada tiene un comportamiento similar a la red aleatoria que representa a las mezclas homogéneas.

\section{Discusión}

Al considerar que se realizan los contactos de manera local y a distancia dentro y entre familias, respectivamente, el tamaño de la epidemia calculado a través de la topología de red del mundo pequeño generalizada se aproxima a la de mezclas homogéneas $\left(\mathrm{V}_{\mathrm{r}}\right)$, desde 2 hasta $30 \%$.
En este trabajo se utilizó la red del mundo pequeño generalizada para representar la estructura de la población. Esta red tiene cierta similitud con la red del mundo pequeño, ${ }^{32}$ en la que inicialmente se propagaba más rápido la información y se obtenía mayor potencia computacional. ${ }^{36}$ Las variantes fueron: a) no se desconectaron los vértices para tener mezclas homogéneas dentro de las familias; ${ }^{26}$ b) se unieron aleatoriamente los vértices, de tal manera que algunos se encontraban cerca o distantes de ellos, lo que comúnmente ocurre en los sitios de reunión; y c) el modelo empleado fue estocástico en lugar del determinístico.

La RMPG es más eficiente cuando el número de ligas por vértice es mayor, por ejemplo cuatro. En el estudio de redes científicas se encontró que en la comunidad de físicos y científicos computacionales, el tamaño de la red es de cuatro y nueve, respectivamente. ${ }^{28,39}$

La red del mundo pequeño generalizada es aproximadamente igual de eficiente que la red del todo aleatoria, ${ }^{41,42}$ al obtenerse valores de L cercanos a 3.5. Era esperable que el valor de la red aleatoria fuera igual a 1 debido a que se asume que se tienen mezclas homogéneas.

En este trabajo se mostró que los sitios de reunión son un factor importante en la diseminación de una enfermedad infecciosa..$^{5}$ El resultado coincide con el obtenido en las medidas realizadas en las escuelas de Hong Kong, en la aplicación de vacunas para la inmunización

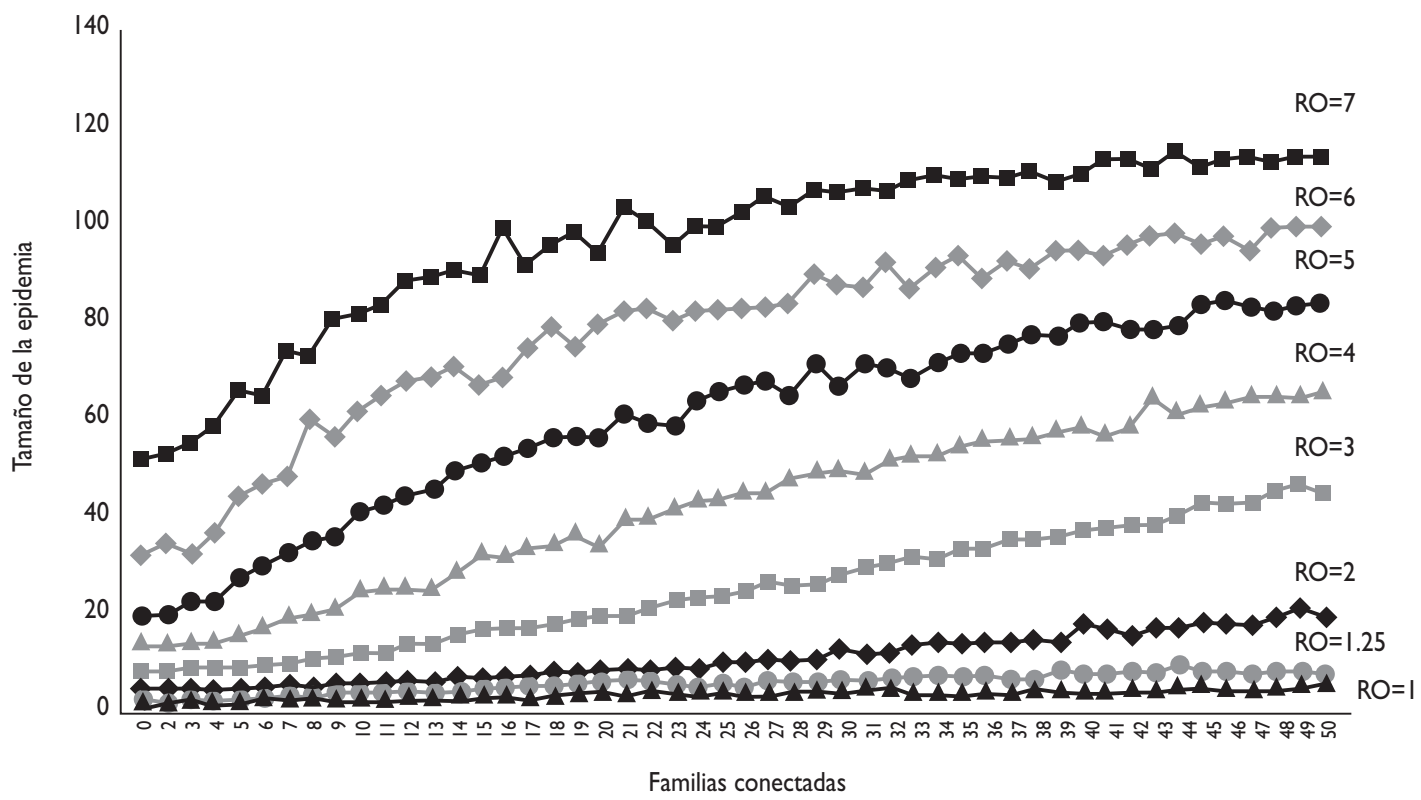

Figura 2. Tamaño de la epidemia al considerar un sitio de reunión, con 50 familias y cinco integrantes. Colima, MÉXICO, 2004. 


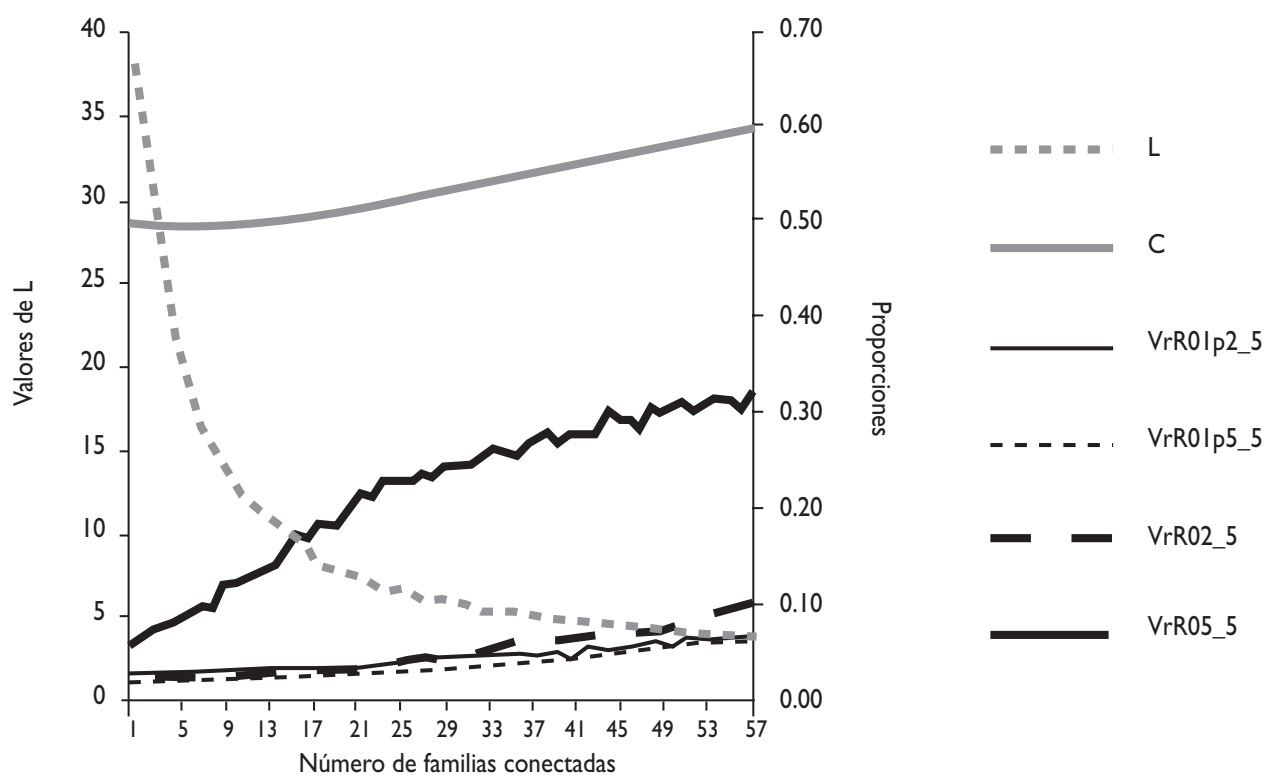

Figura 3. Comportamiento del tamaño de la red (L), Coeficiente de AGrupación (C) Y Proporción de epidemias $\left(\mathbf{V}_{\mathbf{r}}\right)$ CON RESPECTO AL NÚMERO dE faMiLIAS CONECTADAS, PARA UNA POBLACIÓN DE 300 PERSONAS CON 60 faMilias de taMAÑo Cinco. Colima, México, 2004

contra la influenza. ${ }^{10}$ Esto indica que las medidas operativas en el control o erradicación de las enfermedades infecciosas se deben realizar principalmente en los sitios en los que interactúan las personas por un cierto periodo de tiempo.

Con base en los resultados obtenidos en el presente estudio, se concluye que la topología de red del mundo pequeño generalizada fue tan eficiente como una red aleatoria, a medida que interactuaban más familias en los sitios de reunión; y el tamaño de la epidemia se incrementó hasta $400 \%$ conforme interactúan más familias a través de la asistencia a los sitios de reunión. Los factores que influyeron en el tamaño de la epidemia fueron los sitios de reunión, el tamaño de la familia y los valores de $\mathrm{R}_{0}$.

\section{Referencias}

I. Cohen M. Changing patterns of infectious disease. Nature 2000;406 (6797):762.

2. Binder S, Levitt A, Sacks J, Hughes J. Emerging Infectious Diseases: Public Health Issues for the 2Ist Century. Science 284(54I8): I3II.

3. Kay KM. Global defense again the infectious disease threat. Communicable diseases 2002. Geneva, Switzerland: WHO, 2003.

4. Nåsell I. Stochastic models of some endemic infections. Mathematical Biosciences 2002;179(I):1-19.
5. Ball F, Lyne O. Optimal vaccination policies for stochastic epidemics among a population of households. Mathematical Biosciences [serie en internet]. (2002, May), [Consultado 2009 junio 7]; 177/I78:333.

6. Becker N. The uses of epidemic models. Biometrics 1979;35:295-305.

7.Altmann M. Susceptible-infected-removed epidemic models with dynamic partnerships.J Mathem Biol 1995;33:661-675.

8. Heesterbeek JAP, Dietz K. The concept of $\mathrm{R}_{0}$ in epidemic theory. Stat Neerl 1996;50:89-II0.

9. Wallinga J, Edmunds WJ, Kretzschmar S. Perspective: human contact patterns and the spread of airborne infectious diseases. Trends Microbiol 1999;7(9):372-377.

I0. Longini I, Koopman J. Household and community transmission parameters from final distributions of infections in households. Biometrics 1982;38:I I5-I26.

II. Keeling M, Grenfell B. Disease extinction and community size: Modeling the persistence of measles. Science 1997;275:65-67.

I2. Ball F. Stochastic and deterministic models for sis epidemics a population partitioned into households. Mathem Biosc 1999;156:41-67. I3.Allen L, Burgin A. Comparison of deterministic and stochastic SIS and SIR models in discrete time. Mathem Biosc 2000;163:I-33.

14. Chick S,Adams A, Koopman J.Analysis and simulation of a stochastic, discrete-individual model of STD transmission with partnership concurrency. Mathem Biosc 2000;166:45-68.

15. Duerr HP, Dietz K. Stochastic models for aggregation processes. Mathem Biosc 2000; 165:135-145.

16. Hyman J, Li J.An intuitive formulation for the reproductive number for the spread of diseases in heterogeneous populations. Mathem Biosc 2000;167:65-86.

17. Müller J, Kretzschmar M, Dietz K. Contact tracing and deterministic epidemic models. Mathem Biosc 2000;164:39-64.

18. Ball F, Neal P.A general model for stochastic sir epidemics with two levels of mixing. Mathem Biosc 2002;180:73-102. 
19. Kuperman M,Abramson G. Small world effect in an epidemiological model. Phys Rev Lett [serie en internet]. 86(I3):2909-2912. [Consultado 2009 junio5]. Disponible en: http: //guillermoabramson. I I Omb.com/paper/ smallworld/swepi.pdf

20. Lefèbre C, Picard P. Collective Epidemic Models. Mathem Biosc 1996;|34:5|-70.

21.Watts D. Small worlds: The dynamic of networks between order and randomness. EUA: Princeton University Press, 1999.

22. Boots M, Sasaki A. 'Small worlds' and the evolution of virulence: Infection occurs locally and at a distance. Proc Roy Soc London B 1999;266:1933-1938.

23. Kretzschmar M, Morris M. Measures of concurrency in networks and the spread of infectious disease. Mathem Biosc 1996;133:165-195.

24. Tu Y. How robust is the Internet? (Cover story). Nature 2000;406:353-382.

25. Aguda B, Goryachev A. From Pathways Databases to Network Models of Switching Behavior. PLoS Computational Biology [serie en internet] 3(9):1674-1678. [Consultado 2009 junio 7]. Disponible en: http: //people. mbi.ohid-state.edu/baguda/Agudalab/myPAPERS/2007-PLOS-CB.

26. Jasch F, Blumen A. Target problem on small-world networks. Physical Review. E, Statistical, Nonlinear,And Soft Matter Physics [serie en internet]. 63(4 Pt I):04I I08. [Consultado 2009 junio 5]. Disponible en: http: //link.aps.org/doi/I0.I I03/PhysRevE.63.04II 08.

27. Latora V, Marchiori M. Efficient behavior of small-world networks. Phys Rev Lett [serie en internet]. 87(19): 19870I. [Consultado 2009 junio 5]. Disponible en: http://www.ct.infn.it//labra/efficiency_pri_PRL87.pdf 28. Newman M. The structure of scientific collaboration networks. Proceedings of the National Academy of Sciences of the United States of America 200I;98(2):404-409.

29. Lloyd A. How viruses spread among computers and people. Science 2001;292:1316-1317.

30. Koopman J, Chick S, Simon C, Riolo C, Jacquez G. Stochastic effects on endemic infection levels of disseminating versus local contacts. Mathem Biosc 2002; |80:49-7|.

3I. Fell D,Wagner A. The small world of metabolism. Nat Biotechnol 2000; I8:II2I-II 22.

32. Watts D, Strogatz S. Collective dynamics of "small-world" networks. Nature 1998;393:440-442.
33. Ball P. Science is about networking. Nature News [serie en internet] 200I. [Consultado 2008 diciembre 02]. Disponible en: http://www.nature. $\mathrm{com} /$ news $/ 200 \mathrm{I} / 0 \mathrm{I} 0 \mathrm{I}$ /8/full/news010I I8-2.html.

34. Collins J, Chow C. It's a small world. Nature 1998;393:409-4I0. 35. Kleinberg J. Navigation in a small world. Nature 2000;406:845. 36. Hong H, Choi MY, Kim B. Synchronization on small-world networks. Physical Review. E, Statistical, Nonlinear,And Soft Matter Physics [serial on the Internet]. (2002, Feb 24), [cited June 5, 2009];65(2 Pt 2):026।39. 37. Montoya J, Solé R. Small world patterns in food webs. JTheoret Biol 2002;214:405-4I2

38. Xin-She Y. Chaos in small-world networks. Physical Review. E, Statistical, Nonlinear,And Soft Matter Physics [serie en internet]. 63(4 Pt 2):046206. [Consultado 2009 junio 5]. Disponible en: http: //link.aps.org/ doi/ro.no3/PhysRevE.63.046206.

39. Davidsen J, Ebel H, Bornholdt S. Emergence of a small world from local interactions: Modeling acquaintance networks. Phys Rev Lett [serie en internet]. 88(I2): 12870I. [Consultado 2009 junio 5]. Disponible en: http: //www.//p.uni-bremen.de/complex/pr I2870I.pdf.

40. Lago F, Huerta R, Corbacho F, Sigüenza J. Fast response and temporal coherent oscillation in small-world networks. Phys Rev Lett 2000;84(I2):2758-276I.

4I. Mathias N,Gopal V. Small worlds: How and why. Physical Review. E, Statistical, Nonlinear, And Soft Matter Physics [serie en internet]. 63(2 Pt I):02I I I7. [Consultado 2009 junio 5]. Disponible en: http: /leprints.lisc. ernet.in/454/I/Small_worlds.pdf.

42. Sun K, Ouyang Q. Microscopic self-organization in networks. Physical Review. E, Statistical, Nonlinear, And Soft Matter Physics [serie en internet]. ;64(2 Pt 2):026I II. [Consultado 2009 junio 5]. Disponible en: http: //www.soporteuv.mx:2 | 26/ehost/pdf?vid=8\&hid=9\&sid=8bc15 I 1007 68d2-4c10-82f5-b|93d746d30\%40sessionmgr I I

43. Hernández-Suárez C, Castillo-Chavez C.A basic result on the integral for birth-death Markov processes. Mathem Biosc 1999;161:95-104. 44. Shonkwiller R, Thompson M.A validation study of a simulation model for common source epidemics. Inter J Bio-medical Comp 1986; 19(34):175-194. 